\title{
Tarif attractif pour les assurances du personnel
}

Profitez, vous et votre personnel, des primes actuellement avantageuses

\section{Solutions d'assurances pour votre personnel}

En qualité d'employeur vous êtes obligé d'assurer votre personnel en cas de décès et d'incapacité de gain. Le système suisse des assurances sociales se base sur le concept des trois piliers, mais la coordination de ces trois piliers est très complexe. Les conseillers FMH Insurance Services vous aident à ce que vous et votre personnel trouvent la solution optimale:

- LAA - Assurance accidents obligatoire

- LCA - Assurance collective d'indemnités journalières en cas de maladie

- LPP - Prévoyance professionnelle ( $2^{\mathrm{e}}$ pilier)

\section{Des solutions spéciales uniquement pour les médecins}

Puisque l'offre des toutes compagnies d'assurances peut être librement choisie, il existe des très grandes différences dans les taux des primes. Nous avons examiné un nombre important d'offres et nous avons pu conclure un contrat-cadre attractif avec l'assureur CPT pour les assurances de perte de gain en cas d'accidents et de maladie. Dans le domaine de la prévoyance professionnelle LPP, nous présentons des nombreuses solutions de caisses de pension corporatives qui correspondent aux besoins spécifiques des médecins.

\section{Comparer vaut la peine}

Testez notre offre et comparez-la avec vos polices actuelles, vous profiterez avec nos solutions, vous et votre personnel, de primes plus avantageuses.

\section{Talon réponse}

Prénom / Nom

Adresse

NPA / Lieu

Date de naissance

Téléphone privé / cabinet

Atteignable le plus facilement (heure)

Veuillez contrôler mes assurances du personnel et envoyez-moi une offre comparative. (Prière de nous envoyer une copie de vos polices d'assurances actuellles)

Je désire un conseil personnalisé. Téléphonez-moi.

Je suis intéressé à:
$3^{\text {e }}$ pillier a
O Placement avec garantie
Couverture de la famille
O Protection juridique
O Caisse-maladie
O Responsabilité civile prof. 